\title{
Design Analysis of Corridors-in-the-sky
}

\author{
Min Xue* \\ University of Califormia at Santa Cruz, Moffett Field, CA 94035
}

\begin{abstract}
Corridors-in-the-sky or tubes is one of new concepts in dynamic airspace configuration. It accommodates high density traffic, which has similar trajectories. Less air traffic controllers' workload is expected than classic airspaces, thus, corridors-in-the-sky may increase national airspace capacity and reduce flight delays. To design corridors-in-the-sky, besides identifying their locations, their utilization, altitudes, and impacts on remaining system need to be analyzed. This paper chooses one tube candidate and presents analyses of spatial and temporal utilization of the tube, the impact on the remaining traffic, and the potential benefit caused by off-loading the traffic from underlying sectors. Fundamental issues regarding to the benefits have been also clarified. Methods developed to assist the analysis are described. Analysis results suggest dynamic tubes in terms of varied utilizations during different time periods. And it is found that combined lane options would be a good choice to lower the impact on non-tube users. Finally, it shows significant reduction of peak aircraft count in underlying sectors with only one tube enabled.
\end{abstract}

\section{Introduction}

The US air traffic is predicted to increase by two or three fold by 2025 . The traffic volume will exceed the capability of current airspace infrastructure and significant delays are predicted. Besides the researches that are trying to improve the efficiency of airspace system from tactical perspectives, Dynamic Airspace Configuration (DAC) researches address the problem from a strategic viewpoint by dynamically designing the airspace to accommodate the fluctuating traffic volume. Innovative concepts such as dynamic sector design and Corridors-in-the-sky or tubes have been proposed ${ }^{1}$ to increase the airspace capacity and reduce delays. Tubes can absorb high-density traffic that has similar trajectories. Less workload is expected in tubes than classic airspaces due to the organized traffic flow. If aircraft are assumed to have advanced equipments for self-merging and self-spacing, the associated workload can be negligible.

Many researches have been conducted to find the locations of tube candidates. Alipio et al. ${ }^{2}$ and Yousefi et al. ${ }^{3}$ initially proposed to construct tubes between city-pairs. Sridhar et al. ${ }^{4}$ developed a tube network interconnecting airports in clusters seeded by major airports to impact a significant amount of traffic. Gupta et $\mathrm{al}^{5}$ refined the network based on airport clusters using Mixed Integer Programming (MIP) according to costs. In previous work ${ }^{6}$ a method that combines the Hough transform and Genetic Algorithm (GA) was proposed to find tube candidates based on great circle trajectories. Hering ${ }^{7}$ proposed the "Freeways" because of the different structure of core Europe. The "Freeways" tries to pass through or as close as possible of enlarged major airport areas. But to implement tubes, merely identifying 2D locations is not enough, further operational issues need to be studied. Although Hoffman et al. $^{8}$ analyzed a list of design and operational issues for tubes, it is still at conceptual level.

This paper chooses a tube candidate from previous work. ${ }^{6}$ It then presents analysis of the tube in terms of the spatial and temporal utilization and the impact on remaining traffic, which is measured by the number of crossings between remaining flights and the tube. To assist the analysis, new methods have been developed, such as the space-time map and cell-decomposition method. These methods are found to be helpful and efficient. For instance, the cell-decomposition method can make the crossing detection a hundred time faster than the brute-force method. The analysis results suggest to deploy the tube dynamically and to construct tube with parallel lanes at a few altitudes. Finally, a preliminary benefit analysis has been presented based on the peak aircraft count. Significant reduction of the peak aircraft count has been found in underlying

*Research Scientist, UC Santa Cruz. Mail Stop 210-8. email: Min.Xue@nasa.gov, AIAA member 
sectors by enabling such a tube. The methods of analysis can be extended for tubes in any format and also can be applied for a tube network if necessary.

\section{Tube Model}

In previous work, ${ }^{6}$ a method that combines the Hough transform and GA was developed. Flights were assumed to fly great circle flight trajectories and the schedule was based on the track data on April 20, 2007. A flight was allowed to fly on a tube if it had less than $5 \%$ extra flight distance compared with its shortest path distance. The method was applied to optimize the tube location such that number of tube-attendees was maximized under above constrain. The output tubes were ranked according to the number of attendees. Figure. 1 shows top 60 candidates that can accommodate $44 \%$ or 13015 flights. The colors indicate the number of attendees at different segments and different tubes.

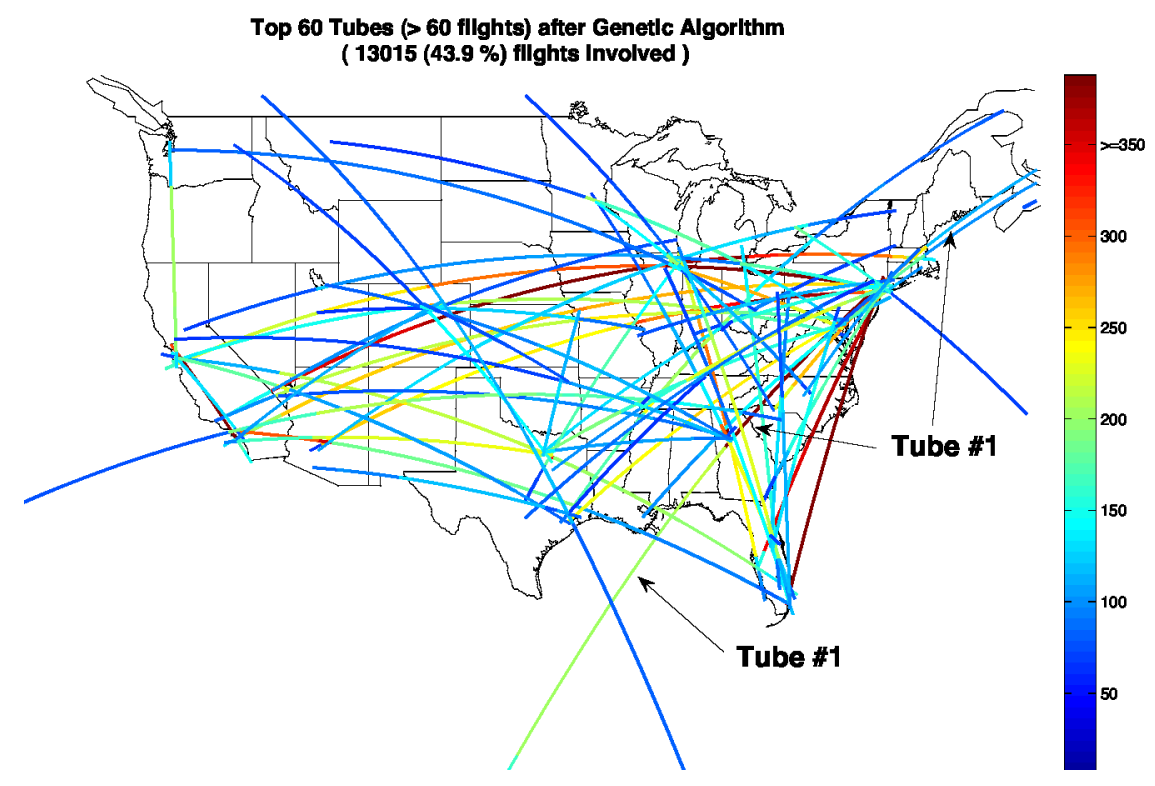

Figure 1. Top 60 tubes ranked by number of attendees

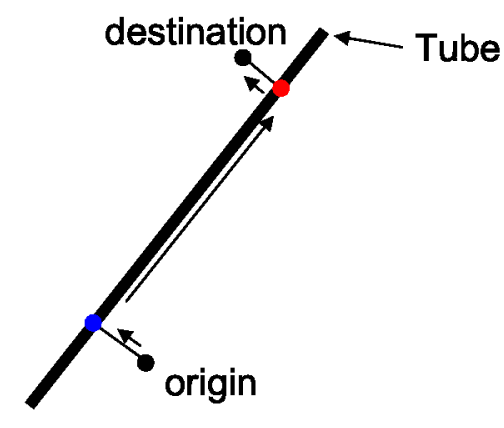

(a) Model of Joining and Exiting a Tube

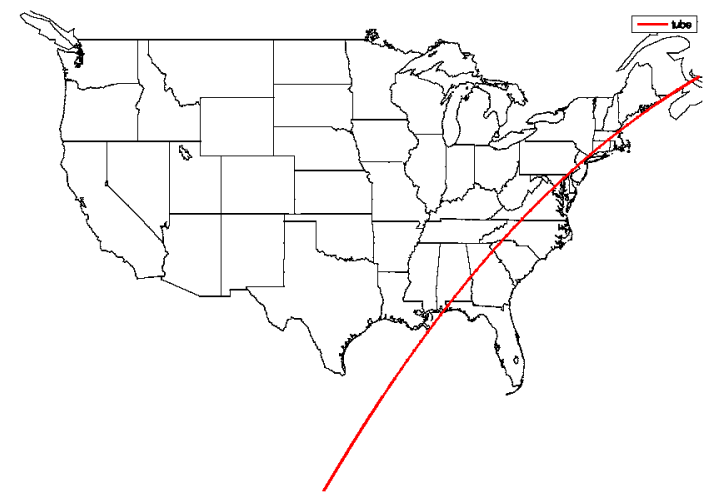

(b) Tube No.1

Figure 2. Tube Model

An attendee was assumed to join in and exit from a tube perpendicularly. The sketch is shown in Figure 2(a). This is the worst case, since other intersection angles will generate less extra distance. Although other model of joining and exiting can be used, as an initial set-up, this model will be used for calculations 
through the paper.

Without loss of generality, the top one candidate (see Figure. 2(b)) is chosen for the purpose of analysis. It has the most attendees, which is 774 flights during entire day. All generated tubes including this one are in great circles. The capacity constraints in airports and the impact of weather conditions are neglected. In following sections, analysis will consist of three parts. The operations in the tube will be examined first. Then the impacts on non-tube users will be analyzed. Finally, benefit of enabling the tube will be discussed and presented.

\section{Operations in Tubes}

There could be many ways for measuring utilization of a tube, such as the number of attended flights. But they may not be good enough to answer such questions: How crowd the flights in the tube would be? What is the minimum number of lanes needed in the tube? To answer these questions, the utilization has to be analyzed in temporal and spatial manner. Therefore, a space-time map is developed based on the space-time diagram used in researches of ground transportation ${ }^{9} .{ }^{10}$

\section{A. Space-time Map}

To generate a space-time map, a spatial scale is needed. An origin is first defined at one end of the tube. Then points on the great circle tube are coordinated according to their distances to the origin. As shown in Figure. 3(a), the upper end is the origin and the scales along the tube are corresponding distances (in nautical miles) to the origin.

Next, a space-time space is set up. As in the space-time diagram, for each flight, one can draw a curve in such a space in terms of its schedule, entry and exit points, and speed. If the speed of the flight is assumed to be constant, the curve becomes a straight line whose slope is the speed. Figure. 3(b) shows the space-time space and a curve corresponding to a flight. From the curve we can tell that the flight joined the tube somewhere around Atlanta at $8 \mathrm{AM}$ and exited somewhere around New York at $10 \mathrm{AM}$. Then, this space is descretized into grids. Each grid has 10 nautical miles in width and 2 minutes in height. A grid can be looked on as a safety zone, which means only one flight is allowed in it. If a curve intersects a grid, the grid will be accumulated by one. Figure. 3(b) shows the grids that are intersected by the sample curve.

Although in this study, the speed of a flight in the tube is assumed to be constant, it is applicable to have flights with varied speeds when necessary. Meanwhile, uncertainty of the schedule, speed and entry location can be taken into account to generate a probabilistic space-time map for tube operations.

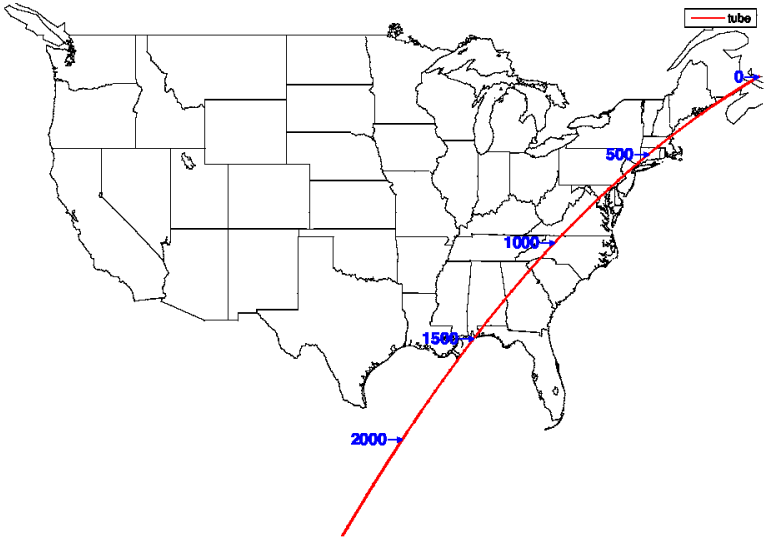

(a) Tube with Scale

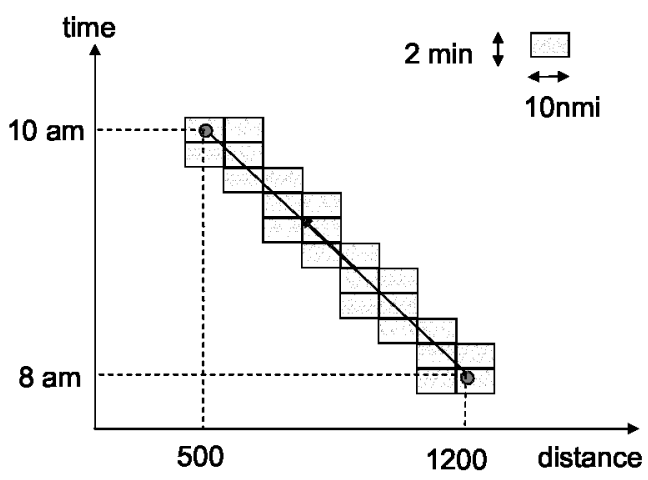

(b) Sketch of Space-time Map

Figure 3. Space Time Map 


\section{B. Analysis of Operations}

Using the method described above, the space-time map of 774 flights in the tube is generated. Typical speeds of different aircraft types are chosen. Entry and exit locations are computed following the rule of "entry and exit perpendicularly". And the flight schedules are used as bases for time. The resulting map is shown in Figure 4. As described in last section, each grid accumulates the times occupied by flights. High pixel value of a grid means high occupancy, which is represented by hot color. Otherwise, it will be filled in cold color. From the figure, one can tell the hottest grids have 7 flights showing up simultaneously. Considering the separation rule, at least 7 lanes might be needed for these spots.

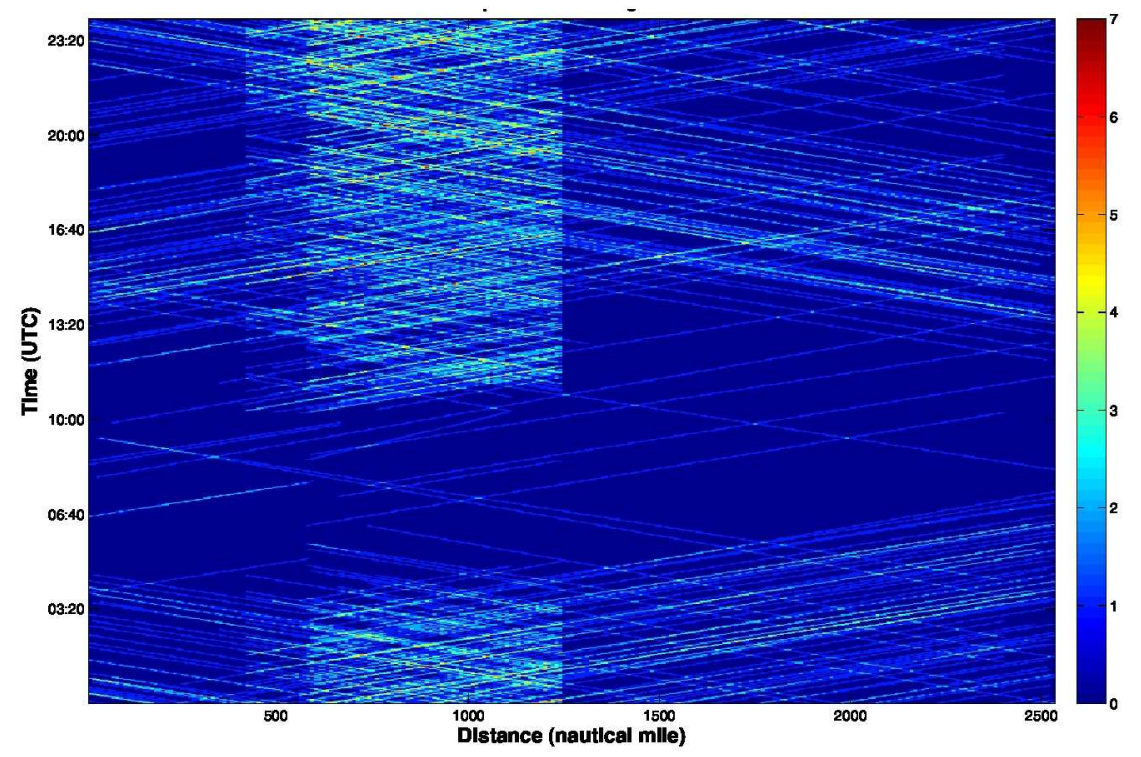

Figure 4. Space Time Map for Tube No. 1

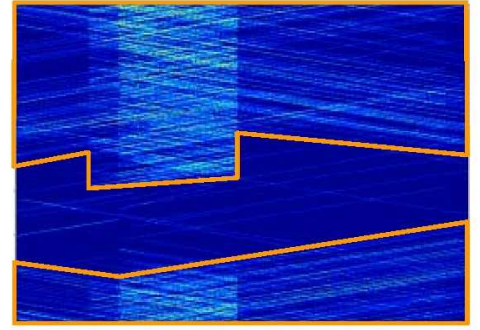

(a) Region for Constructing a Tube

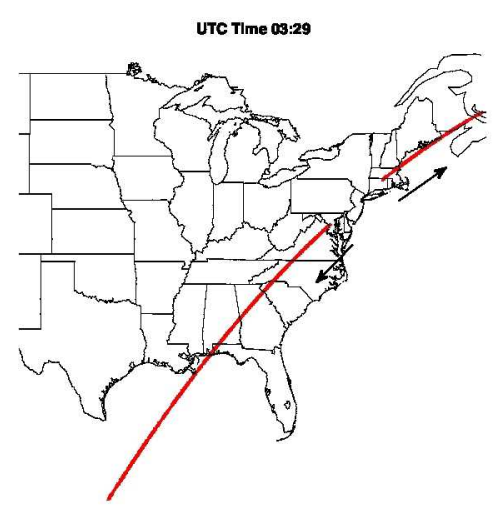

(b) Snapshot 1

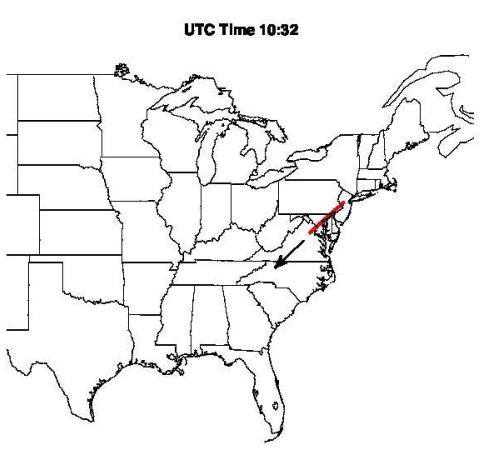

(c) Snapshot 2

Figure 5. Snapshots of Dynamic Tube

In Figure. 4, there are only a few flights in the dark blue region which is roughly between UTC time 4 AM and $10 \mathrm{AM}$. Meanwhile, it is noticed that heavy traffic happens in the brightest region between scale $500 \mathrm{nmi}$ and 1,250 $\mathrm{nmi}$ in daytime. Obviously, it may be not necessary to construct a tube for the dark blue region if there is any undesired cost associated with the tube. Then it brings up the concept of dynamic tube, which is that a tube will be opened to follow the first flight of heavy traffic and ended with the last flight of heavy traffic. By setting up a threshold for triggering a tube, a region in the space-time map can be defined. Then a tube can be built up according to the region. Figure 5(a) shows a sample region included in orange frames in the space-time map. This region is specified for constructing a dynamic tube. Next a 
tube is built up dynamically to follow this framed region. Around 3:20 AM the full length tube will be shrunk gradually. It starts from the location at $500 \mathrm{nmi}$ towards to two ends. At $6 \mathrm{AM}$ the tube will be totally deactivated. At $10 \mathrm{AM}$ the tube will be gradually activated again. It will starts from the location at $500 \mathrm{nmi}$ towards two ends. Around 2 PM, the entire tube will be fully activated. Figure 5(b) presents snapshots of the dynamic tube.

To examine the space-time distribution of entries and exits of this tube, Figure 6(a) shows the space-time coordinates of the entries and exits. The blue points stand for entries and the red points stand for exits. It is noted that most of these points happen at several locations. To further check their clustering natures, the histogram in terms of the spatial coordinates is presented in Figure 6(b). It is noticed that along the tube several locations accommodate the majority of the entries and exits. This indicates tube attendees are not joining the tube all over the place and only several ramps may be needed, which may be positive for constructing this tube. These distribution and histogram are helpful when constructing or analyzing ramps.

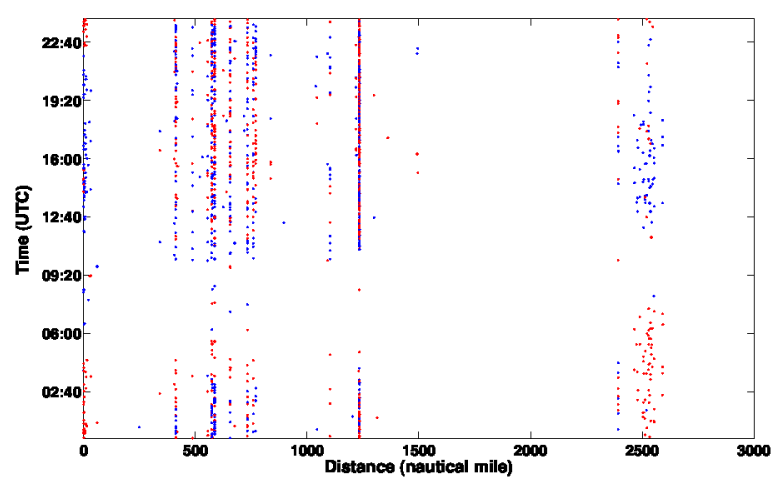

(a) Space-time Distribution

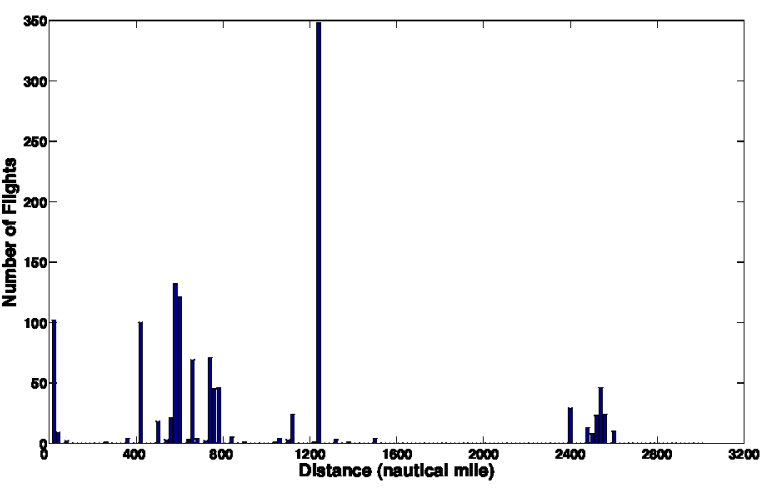

(b) Spatial Histogram

Figure 6. Distribution of Entries and Exits

\section{Impact on Remaining Traffic}

Another import operational issues need to be investigated is the impact on non-tube users. If a significant number of flights are absorbed by tubes, the underlying sectors will have more capacity. But the traffic in underlying sectors will be forced to cap or tunnel if it crosses the impenetrable tube airspace. Therefore, when constructing a tube, a lane option that has less impact on crossing traffic will be preferred. This section will analyze the impact and provide suggestions on lane options for the sample tube. In the analysis, the safety zone is defined as $5 \mathrm{nmi}$ horizontally and $1000 \mathrm{ft}$ vertically.

\section{A. Cell-Decomposition Method for Detecting Crossings}

On April 20, 2007, there are more than 50,000 flights. In the track data, trajectory of a flight is composed of one-minute flight segments, therefore, the number of flight segments are many times more than the number of flights in the daily track data. Since the crossing detection is not a one-time task, significant checks might be expected. For instance, an optimal altitude needs to be found out based on exhaustive search, or the crossing detection may be integrated into optimization for designing tubes. Thus, although brute-force can detect number of crossings in feasible time, a fast or real-time method to detect crossings is desired.

Quad-tree cell decomposition method has been used in robot path planning problems. ${ }^{11}$ It constructs an obstacle-free solution space for building a shortest path with the presence of obstacles. In fact, its theory can be applied in crossing detection problem to rule out most un-related flight segments. To apply quad-tree celldecomposition, a rectangular region which includes entire US continent is defined. The rectangular region is called root cell. The rule of decomposition is that decomposing the cell into 4 quadrants if it contains designated "Obstacles". This decomposition process will be recursively executed until defined depth level is reached. The pseudo code of the cell decomposition is shown in Figure 7.

The following is the procedure for using quad-tree cell-decomposition to detect crossings: 


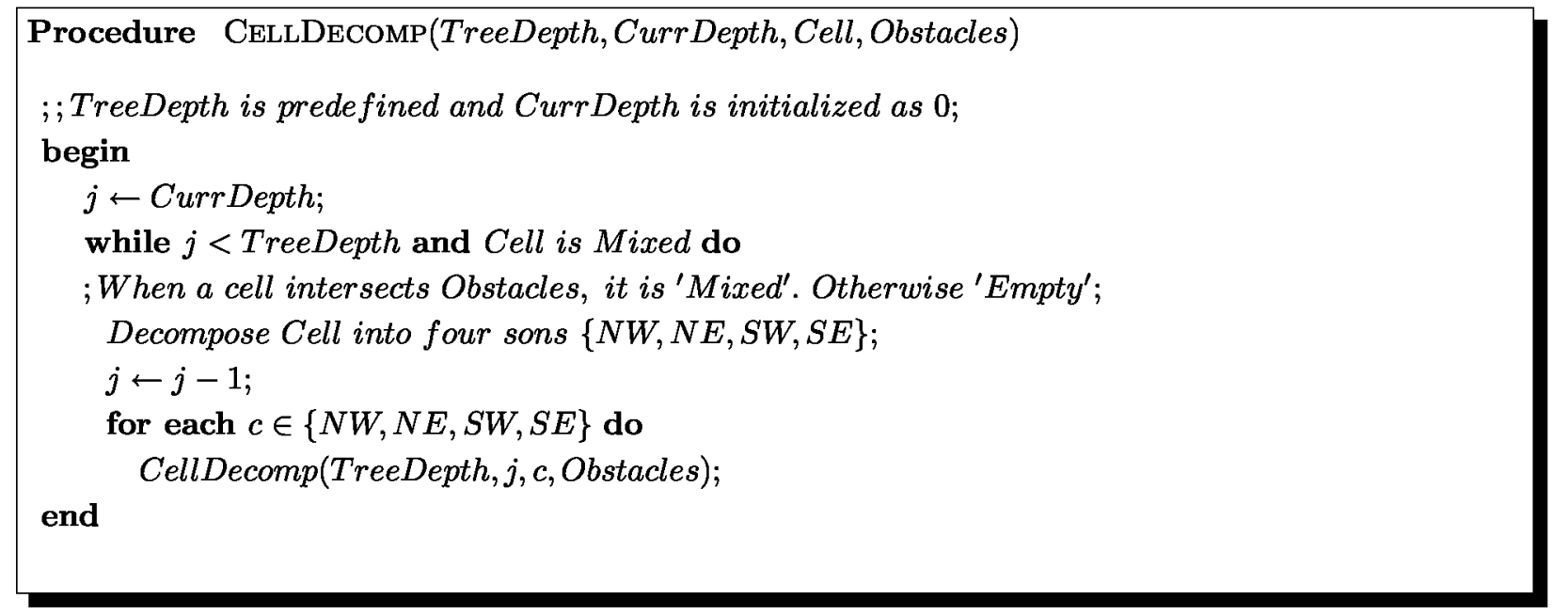

Figure 7. CellDecomp Algorithm

1. Given a depth level and location of a tube.

2. Preprocessing : perform cell-decomposition for all flight segments in entire NAS up to the given depth level. Then every leaf cell is associated with a list of flight segments.

3. Decomposing : perform cell-decomposition for the tube up to the given depth level so that the tube is associated with a list of leaf cells.

4. Checking : For each leaf cells associated with the tube, check its associated flights to see if the flights intersect with the tube. If yes, count the number of distinct flights.

Figure 8(a) displays the decomposed cells for National Airspace System (NAS) traffic with depth level 6. Since the flights are all over the US continent region, most of the cells reached the deepest level. Only a few cells at the left-bottom part are big because there was no flight records. Figure. 8(b) shows the decomposed cells for given tube. It is seen that cells around the obstacles - the tube are small. After this step, only flight segments associated with these small cells will be considered for checking.

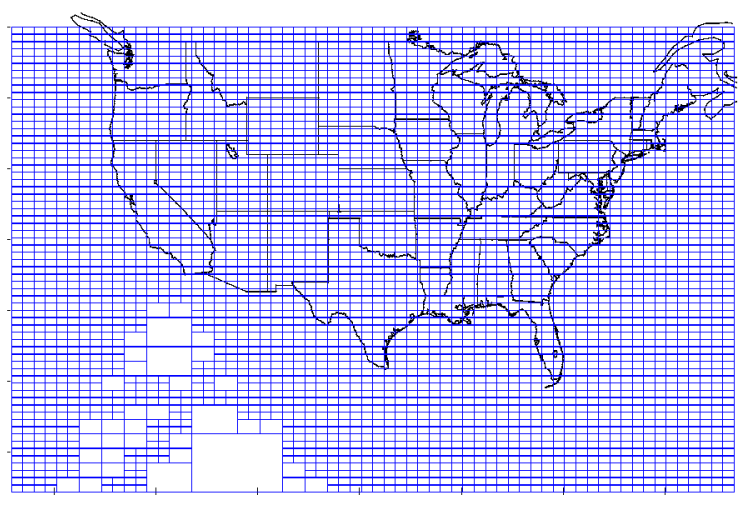

(a) Preprocessing

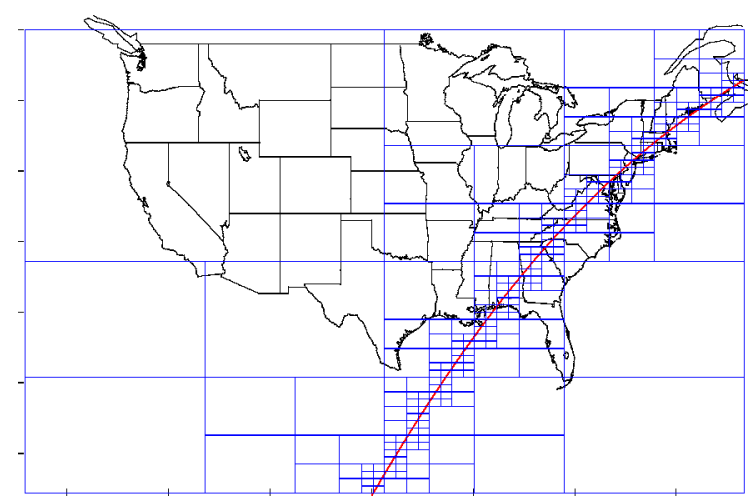

(b) Cell-decomposition of the Tube

Figure 8. Cell-Decomposition for Detecting Crossings 


\section{B. Crossing Detection Performance}

Now the brute-force method is used as baseline. The track data on April 20, 2007 is utilized. All flights above $F L 290$ will be checked. According to the data, there are total 3, 454, 185 flight segments above $F L 290$. The sample case will be : placing the tube at $F L 310$, check the number of crossings between non-tube traffic and the tube. Although the brute-force can take advantage of the altitude range by only considering the flight segments between FL300 and FL320, the number of flight segments is still 205, 222.

The experiments will be run in a Mac machine with a dual core CPU at 2.8GHz. By applying the brute-force method, it takes $8.6 s$ to find the solution. While using quad-tree cell decomposition method, it can takes down to $0.048 \mathrm{~s}$, which is $\mathbf{1 8 0}$ times faster than the baseline. The reason is that brute-force wastes large amount of time on checking flight segments far from the tube. Figure. 9 presents the relative performance when the number of tree depth is increased till 8 . Depth 7 and 8 have the same computational time. It is noted that depth level 8 can not gain any more benefit over depth 7 . That is because the cell sizes at depth 7 is already comparable to the size of one-minute flight segments, further decomposing cells can not rule out more flight segments. Figure. 10 shows the preprocessing time for different depth levels. Since the tree only needs to be built once, the long preprocessing time should be negligible when the detection need to be performed many times. Based on these two figures, depth level 7 should be recommended when performing cell-decomposition for detecting crossings. With the computation time of $0.048 \mathrm{~s}$, this method can be incorporated into the optimization to find good tube candidates that also minimizing crossings of non-tube traffic.

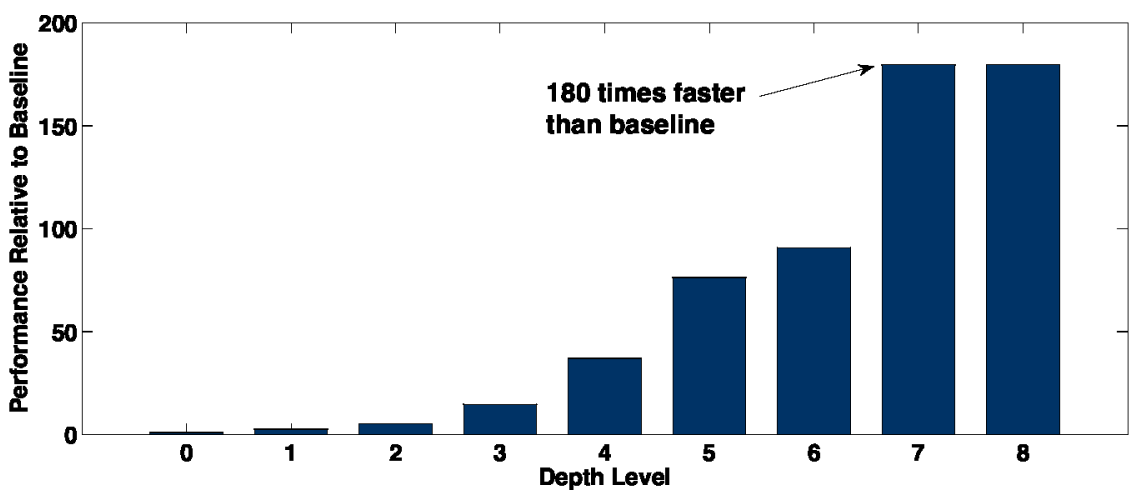

Figure 9. Relative Performances of Crossing Detection

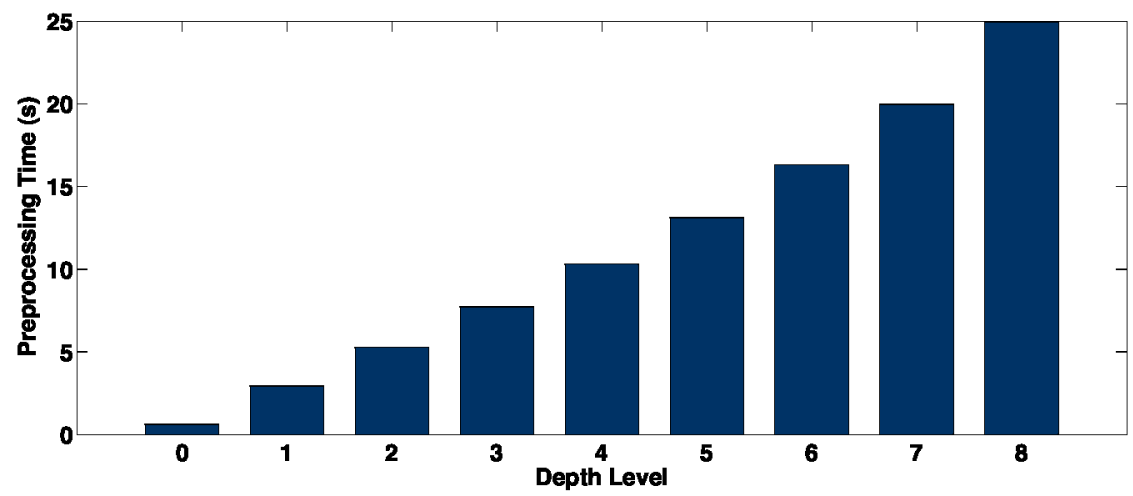

Figure 10. Preprocessing Time

\section{Lane Options}

From the discussions in fist part of this paper, it is noted that at least 7 lanes might be needed in terms of tube traffic with no constraints at airports. Since tubes are expected to be impenetrable, it is desired to find 
out a lane option such that the crossing flights could be lowered. The cell-decomposition method described above will be used.

In this section, middle part of the tube from the location of $500 \mathrm{nmi}$ to the location of $1250 \mathrm{nmi}$ will be studied. To lower the ambiguity, three lane options are predefined. Figure. 11(a) shows stacked lanes by putting 7 lanes vertically. Figure. 11(b) displays side-by-side lanes. And Figure. 11(c) are the mixed option with 3-lane part at a altitude and 4-lane part at another altitude.

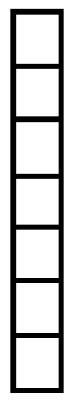

(a) Stacked Lanes

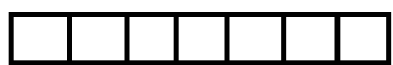

(b) Side-by-side Lanes

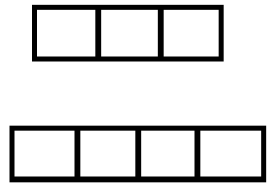

(c) Combined Lanes

Figure 11. Lane Options

Figure. 12 presents the comparison among these three lane options. The "Stacked" Lanes are shown as horizontal lines because they cover 7 altitudes with each lane occupying one altitude. The "Combined" Lanes are a bit difficult for display. With the entire line of "combined lane 1", the three-lane part is fixed at $F L 410$ while four-lane part's altitude is shown as triangles. So do the others. The lines of "combined lane 2", "combined lane 3", and "combined lane 4" has three-lane part fixed at FL350, FL340, and FL290, respectively.

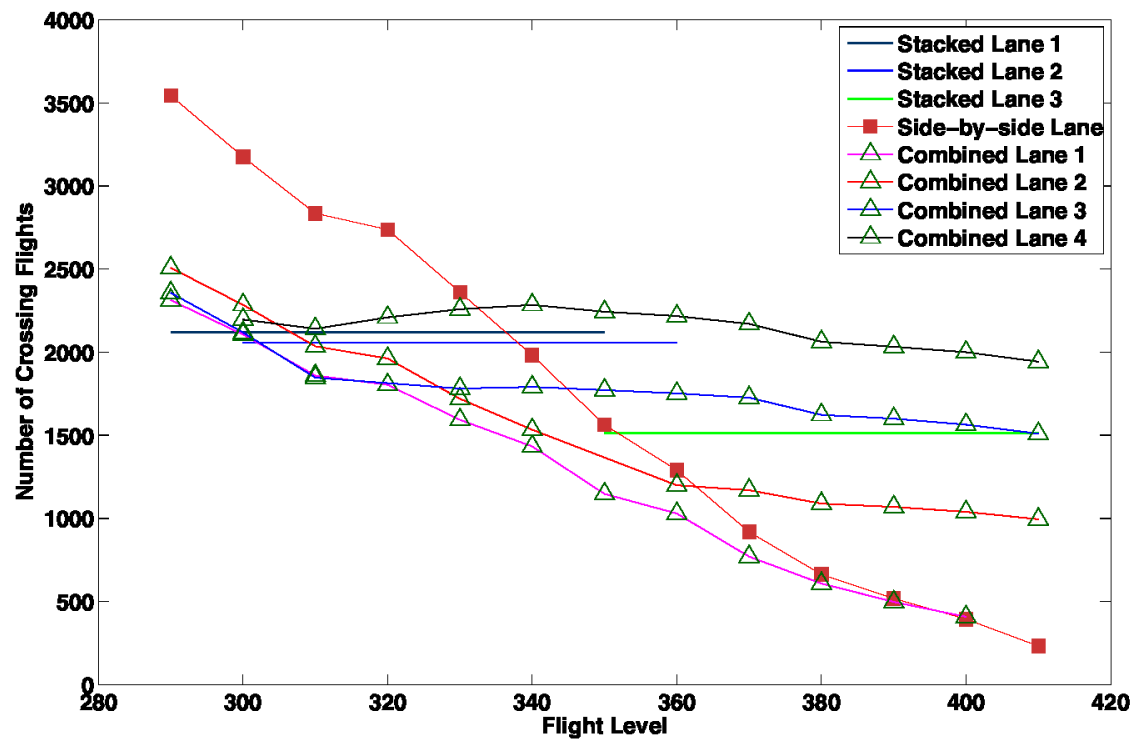

Figure 12. Comparison Among Different Lane Options

Contradicting one's intuition, except at super high altitudes, the side-by-side lane option is the worst according to the number of crossing flights. Although it has low crossings at super high altitudes, these altitudes may not feasible for all tube users. The stacked lane options have relative low crossings, but since they block seven consecutive altitudes, this option may cause high cost for non-tube traffic to cap and tunnel. The combined options that have one part at high altitude and another at low altitude seems attractive. It 
has relative low number of crossings meanwhile it provides tube users flexibility of choosing two different altitudes. Although, these don't serve as final analysis, they provide us insights of the impact on remaining traffic. And there is big differences of the impacts if different lane options are chosen.

\section{Initial Benefit Analysis}

After analyzing utilization and crossings, this section revisits the tube concept and benefits of tubes. According to the proposal, ${ }^{1}$ one possible advantage of having tube airspace is to increase overall airspace capacity, therefore this section will focus on discussing the benefit of increasing capacity. Before discussion, some issues need to be clarified.

Lemma V.1. If tube airspace could increase the capacity of entire NAS then the complexity of handling the same amount traffic in tubes have to be less than classic sectors

Proof. Suppose total number of aircraft operated in a sector is $N$. If one tube is introduced and the tube will accommodate $n$ aircraft, then the underlying sector will handle $N-n$ aircraft. Assume the complexity of managing $N$ aircraft in the sector is $C s(N)$, the complexity of managing $N-n$ aircraft in the sector would be $C s(N-n)$. And the complexity for the tube to manage $n$ aircraft is $C t(n)$. If there is no tube enabled, the complexity $C(N)$ is:

$$
C(N)=C s(n)+C s(N-n)+C s(\text { interaction }(n, N-n))
$$

Where last term on the right side is the complexity caused by the interaction between the group of $n$ aircraft and the group of $N-n$ aircraft. After the tube enabled, the complexity is:

$$
C^{\prime}(N)=C t(n)+C s(N-n)+C s^{\prime}(\text { interaction }(n, N-n))
$$

Where the third term on the right side is the interaction between the tube traffic and the sector traffic. It has two possible values: $C s($ interaction $(n, N-n))$ when the tube is penetrable or porous; Or less when the tube is impenetrable since only crossing traffic in the sector need to be managed. Hence, taking the worst case $C s^{\prime}($ interaction $(n, N-n))$ can be approximately equal to $C s($ interaction $(n, N-n))$. In order to make sure $C^{\prime}(N)$ is less than $C(N)$, the following has to be true:

$$
C t(n)<C s(n)
$$

Since flights inside of tubes are aligned and organized, the de-confliction related workload will be lower than in classic sectors. In the future, if advanced equipage is integrated, further reduction of workload for tubes can be expected. Of course, formal approve or simulation need to be done in future work to examine if it is true with tube operations. The purpose of this lema is to point out there is such a critical assumption regarding to the tube benefits. This should be proved in future's research.

Corollary V.2. Suppose the benefit and cost of a tube are $B$ and $P$ as defined in Eqn. 4 and 5, where Cs(n) is the complexity of handling $n$ aircraft in a traditional sector, $C t(n)$ denotes the complexity of handling $n$ aircraft in a tube, and Cost $(N-n)$ represents the cost of capping or tunnelling $N-n$ aircraft in the traditional sector, the number of aircraft has to be greater than some threshold as in Eqn. 6 in order to make the tube beneficial.

$$
\begin{gathered}
B(n)=C s(n)-C t(n) \\
P(N-n)=\operatorname{Cost}(N-n) ; \\
B>P, \quad \text { when } n>T
\end{gathered}
$$

Proof. Given a constant $N$, since $B(n)$ can be assumed monotonically increasing or decreasing with $n$. And $P(N-n)$ will be inverse proportional to $n$. There should exist a value $T$ such that when $n$ is greater than $T$, the tube is beneficial. Of course, the $T$ will be $N$, if $P$ can never be compensated by $B$. Or it can be 0 if $P$ can be confirmed negligible. 
Obviously, in above corollary, the benefit and cost are still in general format and need to be well-defined in the future, so does the $T$. The purpose of this corollary is to clarify the relationship between the benefit and the cost of the tubes when discussing the tube benefits.

Then, as a preliminary study, the benefit and cost will be discussed based on the reduction of the peak aircraft count and the number of crossings, respectively. From the analysis in above sections, it is known if the tube mentioned above is enabled, the number of crossings would be around 2,000 , which is dependent on the lane profile.

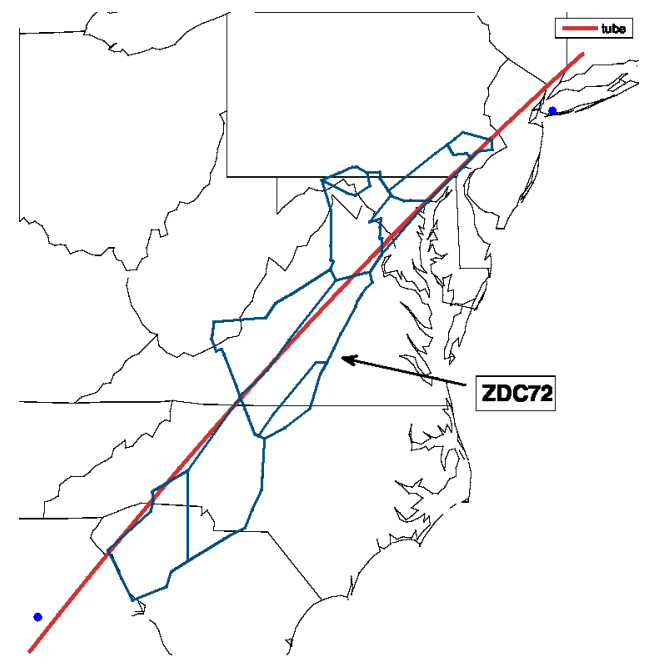

Figure 13. Underlying Sectors

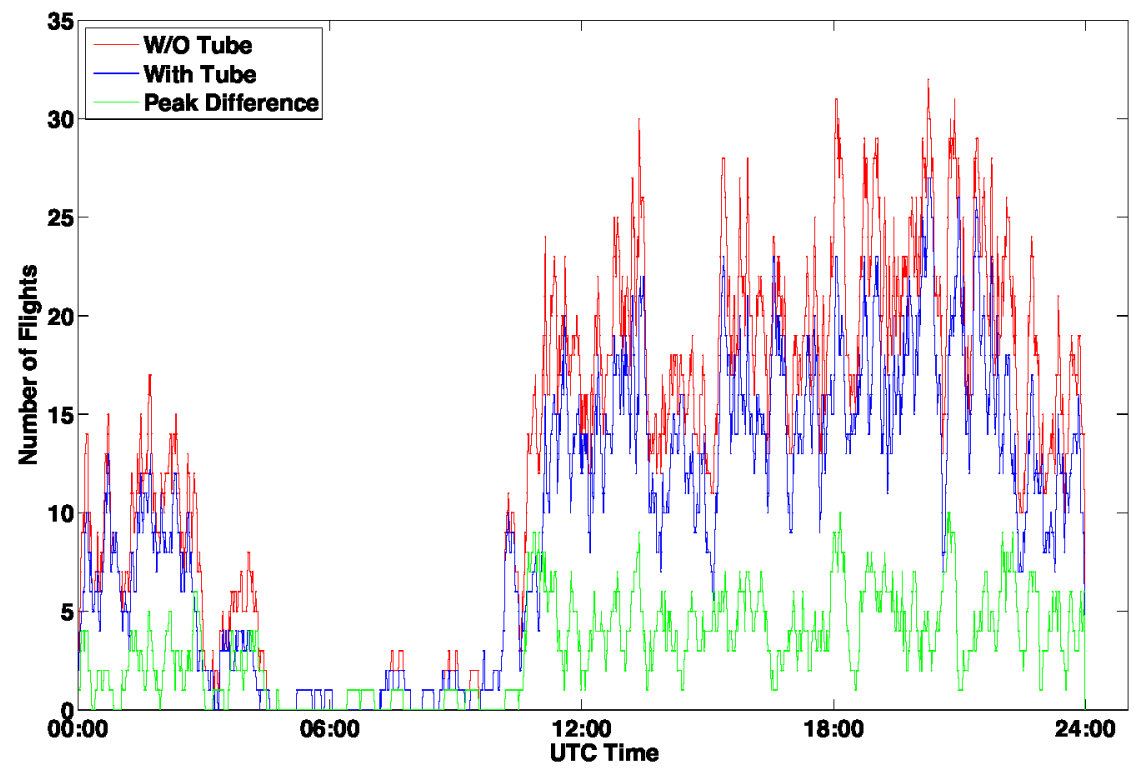

Figure 14. Peak Aircraft Count in ZDC72

Then the peak aircraft count will be used as an approximated measurement of complexity. The tube and its underlying sectors are shown in Figure. 13. The peak aircraft count is first examined for the sectors without the tube. Next, to study the peak aircraft count of sectors when the tube is enabled, the tube traffic were simply removed from the sectors. The time history of the peak aircraft count of ZDC72 with and without tube are shown in Figure 14 in a blue curve and a red curve, respectively. The difference of these two are shown in a green curve. It is noted that the difference of traffic has coincided peaks with overall traffic (blue curve) in ZDC72. Thus, the average reduction of the peak aircraft count due to enabling of the 
tube is as high as $26.1 \%$. In fact, similar situations happen in other sectors with this tube enabled. The results show that Sector ZDC04, ZTL28, and ZNY09 have $17.4 \%, 27.3 \%$, and $24.1 \%$ reduction of the peak aircraft count, respectively. Thus, although it is not a formal proof, the tube concept seems to be a positive solution to increase the NAS capacity.

\section{Conclusion}

This work focused on the analysis of a given tube. To examine and visualize the utilization, a space-time map is developed. It can be used to guide constructing dynamic tubes and to suggest the minimum number of lanes. Then a quad-tree cell-decomposition is developed to speed up the crossing checks by a factor over one hundred. This also enables minimizing crossings in the initial tube design. In the discussion of lane options in terms of minimizing crossings, it is found that combination of multiple lanes at multiple altitudes reduces the number of crossings and provides flexibility for tube users. The analyses of tube utilization and impact on the remaining traffic is important for an optimal design. They can be either done after initial tube design or integrated into the initial tube design.

Finally, in the analysis of benefits, several issues are clarified first: when handling the same amount of traffic, the tube has to have less complexity compared with the classic sector to increase the NAS capacity; The number of aircraft accommodated by tubes has to be higher than some threshold to make the tube beneficial. These two points untangled the issues with tubes and points out two major concerns that needs to be addressed in future research. Then, preliminary benefit analysis was discussed based on the peak aircraft count and the number of crossings. Results show around $25 \%$ reduction of the peak aircraft count of underlying sectors if only one tube was enabled. And the crossings were about 2,000 flights if a good lane option was used. This benefit analysis provides a general picture for what people may expect from tube airspace. In the future, research will focus on the study of complexity of tube operations either through simulations or via proofs. This will be expected to answer the fundamental question regarding to the tube benefits.

\section{References}

${ }^{1} \mathrm{P}$. Kopardekar, K. Bilimoria, and B. Sridhar. Initial concepts for dynamic airspace configuration. In 7 th AIAA Aviation Technology, Integration and Operations Conference (ATIO), Belfast, Northern Ireland, 18-20 September 2007.

${ }^{2}$ J. Alipio, P. Castro, H. Kaing, N. Shahd, O. Sheizai, G.L. Donohue, and K. Grundmann. Dynamic airspace super sectors (DASS) as high-density highways in the sky for a new US air traffic management system. In AIAA/IEEE Digital Avionics Systems Conference, 12-16 October 2003.

${ }^{3}$ A Yousefi, G.L. Donohue, and L. Sherry. High-volume tube-shape sectors(HTS): A network of high capacity ribbons connecting congested city pairs. In Proceedings of the 23rd Digital Avionics Systems Conference, Salt Lake City, CT, 2004.

${ }^{4}$ B. Sridhar, S. Grabbe, K. Sheth, and K.D. Bilimoria. Initial study of tube networks for flexible airspace utilization. In AIAA Guidance, Navigation, and Control Conference and Exhibit, Keystone, Colorado, 21-24 August 2006.

${ }^{5} \mathrm{G}$. Gupta, B. Sridhar, and A. Mukherjee. Freeways in the sky: Exploring tube airspace design through mixed integer programming. In INFORMS Annual Meeting, Washington, D.C., October 2008.

${ }^{6} \mathrm{M}$. Xue and P. Kopardekar. High-capacity tube network design using the hough transform. In AIAA Guidance, Navigation and Control Conference and Exhibit, Honolulu, Hawaii, August 18-21 2008.

${ }^{7}$ H. Hering. Air Traffic Freeway System for Europe. Technical Report EEC Nore No. 20/05, Eurocontrol Experimental Centre, 2005.

${ }^{8} \mathrm{R}$. Hoffman and J Prete. Principles of airspace tube design for dynamic airspace configuration. In The 8th AIAA Aircraft Technology, Integration, and Operation Conference, Anchorage, Alaska, September 14-19 2008.

${ }^{9}$ M. J. Lighthill and G. B. Whitham. On kinemetic waves. ii. a theory of traffic flow on long crowded roads. Proceedings of the Royal Society of London, 229(1178):317-345, 1956.

${ }^{10} \mathrm{C}$. F. Daganzo. A finite difference approximation of the kinematic wave model of traffic flow. Transportation Research $B, 29 \mathrm{~B}(4): 261-276,1995$.

${ }^{11}$ J.-C. Latombe. Robot Motion Planning. Kluwer Academic Press, 1991. 